\title{
SEPARACIÓN Y REVINCULACIÓN FAMILIAR: EL LUGAR DE LOS VÍNCULOS EN RESIDENCIAS DE PROTECCIÓN
}

Camilo Morales Retamal - Universidad de Chile

Camilo Morales Retamal

Psicólogo Universidad Diego Portales

Magíster en Clínica Psicoanalítica con Niños y Jóvenes, Universidad Alberto Hurtado Coordinador Programa de Estudios Interdisciplinarios en Infancias, Facultad de Ciencias Sociales, Universidad de

\section{Correspondencia:}

Camilo Morales

Av. Ignacio Carrera Pinto 1045, Nuñoa, Santiago, Chile camilomorales@uchile.cl 


\title{
Resumen
}

Este artículo presenta una reflexión y un análisis sobre el problema de la internación de niños y niñas en residencias de protección en Chile. Analiza las prácticas y discursos institucionales que caracterizan el funcionamiento de estos contextos basado en el paradigma de la caridad y problematiza la experiencia de la separación afectiva como un acontecimiento que obstaculiza la promoción y la restitución de los vínculos entre un niño, su familia de origen y la comunidad de pertenencia.

Palabras claves: infancia; institucionalización; caridad; separación afectiva; revinculación familiar

\begin{abstract}
This article presents a reflection and analysis on the problem of the placement of children in residential care in Chile. Analyze the practices and institutional discourses that characterize the functioning of these contexts based on the charity paradigm and problematize the experience of emotional separation as an event that hinders the promotion and restitution of the bonds between a child, his family of origin and the community of belonging.
\end{abstract}

Keywords: childhood; institutionalization, charity; emotional separation; family reintegration 


\section{Introducción}

La situación de internación de niños y niñas en residencias ha sido el reflejo más doloroso de la crisis de las políticas de protección de la infancia en Chile. Instituciones que tienen la tarea de cuidar y promover los vínculos, han actuado con descuido y violencia. En lugar de proteger han vulnerado gravemente derechos fundamentales.

Así lo señalan informes de organismos nacionales e internacionales que confirman la grave situación que se vive dentro de estos dispositivos de "protección” los cuales, pese a las numerosas denuncias y recomendaciones, no han modificado en lo sustancial su forma de funcionamiento.

Se trata de un problema estructural e histórico que se expresa en la existencia de una “violación sistemática de los derechos” (Comité de los Derechos del Niño, 2018) cuya causa está dada por una visión tutelar de la niñez y las precarias condiciones de funcionamiento de la institucionalidad a cargo de la protección de la infancia.

Este escenario, que ha sido descrito como crítico, supone una serie de respuestas que reparen los efectos de la violencia estructural del sistema de protección. Lo anterior requiere no sólo de acciones concretas que modifiquen las prácticas negligentes, sino una comprensión de la situación que permita identificar cómo se producen las resistencias a los cambios.

En esa línea el objetivo de este trabajo es presentar una reflexión y un análisis de las visiones y prácticas institucionales sobre la niñez, que inciden en la promoción y la restitución de los vínculos entre un niño, su familia de origen y la comunidad de pertenencia.

A su vez, se describirá el problema de la separación afectiva como consecuencia de la acción de una medida de protección y cómo a partir de esa situación judicial es posible estructurar un trabajo que, desde un enfoque que respete los derechos y la dignidad de un niño y su familia, se viabiliza un trabajo con foco en la historia y los vínculos. 


\section{El paradigma de la caridad en la internación de niños y niñas en Chile}

La internación en residencias de protección es la expresión del modo en cómo nuestra sociedad ha asumido la tarea de cuidar a la infancia vulnerada en sus derechos, pero también da cuenta de la forma en que las instituciones conciben su relación con los niños, familias y comunidades que intervienen cuando se han generado situaciones de negligencia y maltrato.

Esta relación debe ser pensada y caracterizada a la luz del fenómeno de la caridad y el asistencialismo como matriz que sigue estando a la base de muchas de las intervenciones que en la actualidad se generan en este campo de trabajo y que, en definitiva, dan prueba de la preminencia del paradigma tutelar que se mantiene vigente en las políticas públicas e intervenciones dirigidas a niños y familias (Aramburu, 2018).

En Chile, a partir de 1758, año de la creación de la primera casa de expósitos en Santiago (Rojas, 2010), las iniciativas de apoyo a la infancia desvalida provienen de las congregaciones religiosas que se instalan en el país integradas inicialmente por misioneros europeos, quienes por provenir de una cultura diferente a la chilena, presentaban una tradición de intervención a la infancia, caracterizada por la validación de una atención cerrada de los niños, de los sentimientos de compasión y piedad como eje de la atención; y de separar a los niños de sus padres por el daño que éstos pudieran provocarles.

Este antecedente inaugural de la protección de la niñez en Chile, ilustra una forma de intervención donde el asistencialismo y la caridad son los elementos que caracterizan el trabajo de las organizaciones que acogen a niños que han sufrido graves vulneraciones en sus derechos.

Estas instituciones no sólo inauguran un modelo particular de política social dirigida al cuidado y a la protección de la infancia, también instalan una visión hegemónica sobre la niñez, la parentalidad, la crianza y la familia. 
Como señala Villalta (2013) históricamente en las sociedades ha predominado una visión de la niñez que ubica al niño en el lugar de la desviación o de la vulnerabilidad. En consecuencia, esta representación instala la idea que se requiere de políticas sociales que intervengan desde una lógica asistencialista y cuyo principal efecto es dejar al niño en una situación objeto de protección de las instituciones.

Bajo esta representación de infancia, el discurso de la caridad deja al niño en un lugar de dependencia y cautividad en relación con un sistema que opera a través de la moralización de sus conductas y lo hace objeto de sus intervenciones. De cierta forma, los niños se vuelven "propiedad" del dispositivo residencial (Bustelo, 2011) que los hace víctimas del despotismo, de la benevolencia y, en consecuencia, los expone a toda clase de abusos toda vez que se le considera un objeto receptor de cuidados dadivosos y no sujetos de pleno derecho.

En este paradigma los sentimientos de caridad y el deseo de proteger a otro en condición de desvalimiento son motores efectivos para identificarse con el sufrimiento de un niño en dificultades. Desde ese lugar, el proceso para implicarse afectivamente será a partir de una carencia que se inscribe del lado del otro (el niño) que ha sufrido una experiencia de vulneración, muchas veces irreparable a los ojos de los equipos residenciales que acogen y deben trabajar con ellos.

Lo anterior genera el fenómeno de la "sacralización del niño" (Levobici y Soulé, 1993) que refiere a la sobreidentificación de los profesionales de la institución con el sufrimiento del niño considerando, sin matices, que los padres son nocivos e incapaces de ejercer adecuadamente sus funciones, por lo que el fantasma de la sustitución se instala tempranamente con la internación.

Esto produce una forma de intervención que supone al niño y/o su familia sin posibilidad de exigir, demandar y tener una participación en el proceso de intervención. La caridad establece 
una relación de poder donde niño y familia deben ser dóciles y estar de cierta forma agradecidos por lo que reciben de parte de un otro con más poder y recursos.

En consecuencia, la caridad opera desde un paradigma tutelar, es decir, en contraposición al paradigma de los derechos humanos y de la protección integral de los derechos de niños y niñas.

Por otra parte, desde el enfoque caritativo la experiencia de separación que sufre un niño en un contexto residencial dejaría una herida que supone la necesidad de una intervención cuyo propósito es entregar al niño a la brevedad una experiencia emocional que sustituya la falta de padres o cuidadores.

Proveer a un niño institucionalizado de una experiencia afectiva que se traduzca en la conformación de un vínculo continuo y permanente es a todas luces un elemento central del trabajo que se debe realizar en estos contextos, pero que no puede efectuarse a priori en detrimento de los padres o familiares (Eliacheff, 2002).

Por el contrario, como se señala en la Convención Internacional de los Derechos del Niño, el Estado debiese entregar asistencia adecuada para que los padres o representantes legales de los niños puedan desempeñar sus funciones de crianza y cuidado (art.18). Lo anterior se reafirma por lo señalado en las Directrices de Naciones Unidas sobre Cuidados Alternativos (2008) al enfatizar la importancia que tiene para un niño mantenerse bajo el cuidado de sus padres o bien de recuperarlo a la brevedad cuando ha sucedido una ruptura: “...los esfuerzos deberían ir encaminados ante todo a lograr que el niño permanezca o vuelva a estar bajo la guarda de sus padres o, cuando proceda, de otros familiares cercanos. El Estado debería velar por que las familias tengan acceso a formas de apoyo en su función cuidadora”. (ONU, 2009, p.2)

Por lo tanto, un nuevo contexto de cuidado y de posibles nuevos vínculos no puede configurarse ser a partir de la sustitución o exclusión de figuras significativas como los padres, 
hermanos u otras personas relevantes en la historia de un niño. Por el contrario, se les debe incluir desde el comienzo, siempre que no exista un resquicio legal que señale lo contrario. El marco de orientaciones y principios que ofrecen los lineamientos del derecho internacional en materia de infancia establecen con claridad que el propósito de la protección residencial no es reemplazar a los padres, sino que generar un trabajo con la familia que permita que ese niño pueda ser cuidado por adultos que garanticen su cuidado y protección.

A lo anterior se debe sumar que existe un mandato desde la Convención y sus Directrices que señalan explícitamente que se debe evitar la separación del niño a través de todos los medios de los que se disponga, buscando siempre alternativas de cuidado familiar para no tener que someter al niño a una situación de separación y menos a un contexto de internación.

En definitiva, una institución debe otorgar condiciones materiales de cuidado y protección dignas, pero también favorecer el establecimiento de contextos que permitan el desarrollo de nuevos vínculos afectivos y, al mismo tiempo, el resguardo y la promoción de los vínculos de origen que todo niño trae consigo independiente de su edad o el tiempo que compartió con su familia antes de la separación.

\section{Familias, residencias y políticas de protección: la fragmentación en las intervenciones}

En otro plano, es necesario reflexionar cómo la existencia de contextos que tienen como tarea acoger a niños que no pueden ser cuidados por sus padres u otros adultos de su red familiar son consecuencia de problemáticas estructurales que remiten a las condiciones de desigualdad y pobreza de una determinada población que no cuenta con el apoyo necesario para el cuidado de sus hijos. Algunas investigaciones señalan que el $77 \%$ de los niños que se encuentran en residencias de protección provienen de familias que viven en situación de pobreza (Universidad Diego Portales, 2018) 
El punto anterior nos parece sustantivo pues la escena de la separación y la internación de niños acontece principalmente en un contexto marcado por la desigualdad, la pobreza y la exclusión. Así lo han señalado instituciones y organizaciones internacionales en los últimos años a través de investigaciones y documentos de trabajo de difusión pública (Comisión Interamericana de Derechos Humanos, 2013; Relaf, 2011; UNICEF, 2002).

Esta condición de vulnerabilidad constante a la que muchas familias están sometidas inevitablemente genera el "desgaste de los lazos familiares" (Anglade, 2005), no sólo por condiciones de vida adversas, sino fundamentalmente porque se produce una experiencia de humillación y frustración que incide en sus relaciones internas y externas.

Los padres, en particular, se encuentran de frente con el fracaso, la sospecha y el cuestionamiento a su rol. En esta condición se genera una experiencia de sentirse permanentemente puestos a prueba que los lleva a la victimización o a adoptar formas de resistencia que son percibidas como un rechazo injustificado hacia el apoyo que brindan las instituciones de protección social. Se califica a estos padres como multiproblemáticos, inhábiles, incapaces y se les trata, en consecuencia, como un cuerpo extraño que hay que modificar o neutralizar a través de dispositivos de gobierno que buscan transformar las conductas del grupo familiar (Grinberg, 2016).

Uno de los efectos más nocivos de esta condición impuesta desde las políticas públicas es que la familia queda atrapada entre la exigencia y la impotencia al verse demandada a responder requerimientos que la exceden en sus posibilidades siendo incapaces de responder, ante los ojos del sistema de protección. Se configura una experiencia de fracaso que es la imposibilidad por allanar un camino de futuro para sus hijos y con ello se produce una deslegitimación de su lugar de padres. De esta forma se da paso a una reacción, por parte de las instituciones de protección, 
que es expresión de una forma de autoridad que ejerce su poder a través del control y la devaluación.

Lo anterior, se complejiza si tomamos en cuenta que la intervención en este campo opera, por lo general, de forma segregada sin posibilidades reales de establecer un trabajo articulado con otros equipos e instituciones. Desde esa perspectiva, las respuestas que se generan a través de las políticas públicas enfocadas al problema de la internación no sólo involucran el desarrollo de acciones en el nivel de la asistencia social y la entrega de beneficios que subsanen las carencias afectivas y materiales de niños y niñas que han sido privados de los cuidados parentales. También es necesario pensar en el nivel de la intervención con la familia de origen, más allá del encuentro que se produce durante una visita o del trabajo que desarrolla el equipo técnico al interior de una residencia. Es necesario buscar soluciones que integren, articulen y permitan dialogar con diferentes actores (Consejo Nacional de la Infancia, 2017)

En este campo de intervención es posible observar permanentemente los efectos que deja en las vidas de niños y padres el funcionamiento fragmentado de las instituciones que forman parte del sistema de protección. Se trata de múltiples formas de invisibilizar, estigmatizar y excluir las experiencias vitales dolorosas de personas que han sido dañadas en su dignidad.

Una forma de ilustrar lo anterior es la contradicción que se configura, desde el sistema protección, sobre el lugar de las familias. Por un lado, se les considera como un factor protector, un recurso para los interventores y otras instituciones de la sociedad, pero también es fuente de vulneraciones, malestar, sospechas y críticas. Se genera así un doble discurso pues, por un lado, se enaltece e idealiza la función de la familia, pero, por otro lado, se cuestiona y descalifica cuando sus miembros no se ajustan al funcionamiento esperado por el orden social. 
El principal efecto que tiene esta paradoja es que los niños quedan muchas veces atrapados entre dos posiciones sobre cómo se entiende el derecho a vivir en familia. Desde un punto de vista, está la situación de la preservación de los vínculos de origen, lo que entra muchas veces en tensión con la situación de vulneración de derechos que generó la separación. Cuando la situación de vulneración fue ejercida sobre el niño por algún familiar cercano se suele resolver esta situación alejándolo del contexto vulnerador. El costo de esta solución es que pierde la continuidad de sus vínculos y es desarraigado de su contexto sin la certeza de que habrá un trabajo que repare ese vínculo.

La incertidumbre para realizar una intervención con un niño y su familia está dada, en parte, por la fragmentación y el desbalance de las instituciones y programas para articular respuestas integrales (Cunill-Grau, 2014). Y es que el trabajo entre instituciones y equipos de intervención presenta numerosos obstáculos que van desde la operatoria para gestionar una reunión o encuentro, hasta la serie de elementos ideológicos que surgen en los procesos de intervención y que evidencian las profundas fricciones en el hecho de comprender y abordar un caso.

Si consideramos relevante avanzar hacia la integración intersectorial e interdisciplinar en el abordaje de casos circunscritos al ámbito de la protección de los derechos de los niños es necesario comenzar haciendo visibles aquellos discursos que están implícitos en el trabajo cotidiano y que actúan como representaciones que encierran prejuicios y exigencias que recaen sobre las personas y los interventores.

Cuando se generan discursos como los que afirman que existen, por un lado, profesionales que defienden los intereses de los niños, mientras que otro grupo se ubica del lado de las familias, establecemos una falsa dicotomía en el sentido de negar que el trabajo con un niño no puede 
prescindir, menos excluir, la esfera de sus vínculos afectivos, de pertenencia y la historia que se ha construido en un contexto y territorio particular.

La fragmentación de la intervención en infancia hace obstáculo a la posibilidad de reflexionar y construir soluciones con otros. Pero también genera una dificultad para escuchar la dimensión del sufrimiento inscrita en las experiencias de las personas que transitan por este sistema. La pregunta en este punto es evidente: ¿Por qué resulta tan complejo escuchar en estas historias el malestar social que da contexto a las dificultades en el ejercicio del cuidado?

\section{La experiencia de separación entre un niño y su familia de origen}

Una de las preguntas que este trabajo busca desarrollar es ¿cómo experimenta un niño la separación de sus padres en un contexto de vulneración de derechos? Si bien la separación y la carencia afectiva han sido objeto de importantes investigaciones entre los años 1940 a 1960 a partir de los trabajos de Donald Winnicott, Rene Spitz y John Bowlby, entre otros, el contexto social y político de esas experiencias es diferente al escenario de nuestras actuales residencias de protección. Una particularidad de la realidad actual de los niños en hogares es la judicialización de las separaciones. Esto quiere decir que los efectos de las rupturas vinculares entre niños y sus padres deben ser analizadas a partir de la intervención que el dispositivo legal realiza en una situación de vulneración de derechos y que tiene como consecuencia determinar la continuidad de la relación entre un niño y sus padres.

Desde ese punto de vista, es un deber ético visibilizar el drama humano vigente de forma cotidiana en las experiencias de niños y padres que se enfrenta a la dolorosa situación de perder la continuidad de sus vínculos en contra de su voluntad y a partir de la acción de un tercero (Morales, 2015). Cuestión que se experimenta muchas veces con violencia e injusticia, sin palabras que permitan recubrir y acompañar una fractura que parece insondable para aquellos la padecen. 
En el caso de niños que han sido privados de sus vínculos primarios la separación es una acción externa, ejecutada por un tercero, que genera una ruptura sobre el lazo que une al niño con un adulto. Esta separación se establece en nombre de la protección del niño en situaciones en donde éste puede quedar expuesto al maltrato o la grave negligencia. Habitualmente no se consideran los efectos subjetivos que la operación de corte genera en el niño, así como en sus referentes primarios.

En definitiva, un niño que ingresa a una residencia de protección no sólo es víctima de la vulneración de sus derechos, sino que también queda expuesto a la pérdida de sus vínculos (Marchant \& Morales, 2016)

Los niños que ingresan a contextos residenciales han sufrido una ruptura en su experiencia vital, una discontinuidad muchas veces desgarradora como consecuencia de la medida de protección y que no es debidamente acompañada e integrada por los contextos residenciales. La separación es un acontecimiento anterior a la internación y, por lo general, anterior al abandono que se produce, en algunas ocasiones y de forma progresiva, en los contextos residenciales (Universidad Diego Portales, 2008).

Se le ha tomado poca atención al fenómeno de la separación y cómo este afecta no sólo a los niños, sino también a sus padres, e incluso a quienes trabajan en contextos de cuidados alternativos. Una institución que no aborda los efectos de una separación limita las posibilidades de apoyar a un niño y su familia en dificultades. Para las instituciones es difícil arreglárselas con la separación, porque es una situación dolorosa que en algún punto es expresión de la injusticia cuando no se han generado condiciones que permitan reconocer y acompañar el malestar subjetivo de los padres y su correlato social.

La separación remite a un acto que proviene de un tercero, no contempla la voluntad de las partes, y se impone sin medir efectos. Las separaciones no se preparan o se acompañan para 
hacerlas comprensibles para niños y adultos. Esta forma de ruptura no solo implica la ruptura de la relación, sino que supone un traumatismo asociado una experiencia que se vuelve difícil de integrar para el niño (Humphreys, 2011)

A su vez, la separación no es un acontecimiento que se pueda trabajar al margen del funcionamiento institucional, requiere del compromiso de la residencia y sus miembros: ¿cómo acoge una institución el dolor de un niño que ha sido vulnerado en sus derechos y a la vez debe enfrentarse a la experiencia de ver interrumpida de forma abrupta la continuidad de sus vínculos?

Una de las grandes dificultades a las que se enfrenta un niño que ingresa a una institución es la ausencia de otro que permita el reconocimiento de un pedido de ayuda particular dirigido hacía él. El funcionamiento del dispositivo institucional va privando, a través de diversos mecanismos, de la posibilidad de que el niño pueda comunicar lo que ha vivido. Lo anterior se agudiza ante la ausencia de una presencia, de un adulto que pueda unificar y darles continuidad a sus experiencias. Trabajar sobre los efectos de la separación implica instalar preguntas por la historia de los niños, por la pérdida de alguien con quien se tuvo un vínculo y por la forma en que esos lazos pueden reconstruirse a través del encuentro con otros.

En síntesis, cuando hablamos del acontecimiento de separación afectiva producido al interior del sistema de protección residencial hacemos referencia a tres dimensiones: una experiencia de discontinuidad de los ritmos y rutinas del niño; una experiencia de extrañamiento y desplazamiento territorial que se produce al salir del contex to comunitario de pertenencia; y una experiencia de desarraigo e injusticia que expone al niño a la pérdida abrupta de su historia e identidad.

La falta de palabras sobre los orígenes, la imposibilidad de tener pertenencias y la omnipotencia y goce de las instituciones que anulan los vínculos de origen, terminan por generar 
efectos devastadores en la subjetividad. Se puede hipotetizar que la separación y los procesos de institucionalización no sólo privan a los niños de las provisiones afectivas (Spitz, 1965). La separación y posterior institucionalización privan total o parcialmente al niño de su memoria al obstaculizar los procesos de transmisión y circulación del deseo.

La historia y el deseo que precede la existencia del niño es fundante en la constitución subjetiva. Por lo tanto, la ruptura que puede llegar a producirse en ese territorio tiene un impacto significativo en el advenimiento del sujeto humano. La separación, desde ese punto de vista, es un acontecimiento que genera un corte en el proyecto identificatorio y de subjetivación del niño (Dolto, 2001).

Como se ha señalado anteriormente, en este campo de trabajo con la infancia se suelen sostener y justificar intervenciones sin referentes significativos, como si estos pudiesen ser intercambiados, sustituidos o anulados a destajo y sin consideración de historia alguna.

\section{Consideraciones para el trabajo de revinculación entre un niño y su familia de origen}

Los grupos familiares intervenidos por los sistemas de protección de la infancia han sufrido transgeneracionalmente condiciones de exclusión y marginación social que afectan las posibilidades del ejercicio de la crianza y cuidado de un niño. Esta característica, que tiende a repetirse, no sólo constituye un dato descriptivo de la situación de la familia, sino que también permite la comprensión de un sufrimiento que no suele ser considerado desde la intervención psicosocial.

Por otro lado, la intervención familiar, en el ámbito de la protección y restitución de los derechos, requiere de un marco social y político que permita una aproximación a la trayectoria que ha tenido el grupo familiar y cómo esos movimientos han afectado su capacidad para vincularse y 
cuidarse mutuamente, así como también el modo que estos pueden relacionarse con las instituciones sociales.

Los contextos de protección residencial no presentan orientaciones claras sobre cómo promover y preservar los vínculos de origen de un niño (Universidad Diego Portales, 2018). Existe más bien un temor y un rechazo de esos lazos de origen en tanto representan el daño, el malestar y el sufrimiento. En su afán por proteger a los niños las instituciones prefieren rechazar esos elementos, encarnados en los padres o la familia, antes que emprender la elaboración de las experiencias de vulneración y violencia incorporando estas figuras que actuaron en desmedro de su propio bienestar.

La situación de los niños separados de sus familias e internados en residencias es una problemática compleja, ya que implica resolver una situación judicial y al mismo tiempo las consecuencias psíquicas que tiene para ese niño y su familia esta nueva situación.

Desde ese punto de vista, es posible plantear que desde que un niño o niña ingresa a una residencia se ve enfrentado a dos vulneraciones. La primera que se relaciona con aquella vivida en su contexto familiar, y la segunda aquella que tiene como consecuencia la separación afectiva de sus vínculos primarios, dicho de otro modo, no sólo se trata de niños y niñas que han sido vulnerados en sus derechos, sino que al mismo tiempo han de vivir una segunda vulneración asociada a las consecuencias psicológicas derivadas de la separación afectiva, cuestión que se denominará como “doble vulneración” (Marchant, 2014).

Como ha sido descrito anteriormente, la separación no es un acontecimiento que afecta sólo en el nivel de la relación particular que une a un niño o niña con su familia, sino que también implica una ruptura en el plano social e institucional. Un niño internado también es desarraigado 
(UNICEF, 2002) de su contex to más próximo y por lo tanto se produce una ruptura con el espacio social y comunitario al cual pertenecía.

Por otra parte, las familias presentan serias dificultades para vincularse a las instituciones, redes y a los profesionales interventores dado que perciben con una gran desconfianza la intervención social en su conjunto.

El trabajo de revinculación implica entonces una intervención que se da en el plano individual, relacional, comunitario y social de los niños y sus familias.

La revinculación familiar es un proceso gradual que implica, en primer lugar, ofrecer al niño un vínculo afectivo que ayude a establecer un sentimiento de seguridad y organizar su vida afectiva que ha sufrido una importante discontinuidad. En segundo lugar, generar acciones que favorezcan la continuidad de los lazos familiares mientras el niño se encuentre separado de su familia en un contexto residencial, así como también llevar adelante acciones que permitan la preparación y acompañamiento para el retorno definitivo, siempre que sea posible y considere el interés superior del niño, a un hogar familiar.

Este proceso requiere como principio que los contextos residenciales mantengan la continuidad de los vínculos entre el niño y su familia, favoreciendo la participación de los padres u otros adultos responsables en la crianza y educación de los niños dentro del contexto residencial. Por último, preservar las pertenencias materiales e inmateriales de los niños o niñas acogidos en el sistema de protección.

Las pertenencias son aquellos objetos que narran o materializan una experiencia significativa en la historia de un sujeto. Rememoran situaciones que se han vivido, recuerdos de vínculos anteriores, que nos invitan a pensar quiénes hemos sido y lo que somos para otros (Marchant \& Morales, 2016) A su vez, estas funcionan como soportes de memoria; son objetos 
que narran lo que fue inscrito en un tiempo anterior, nos hablan de los vínculos significativos y de la historia social de los niños. En conclusión, son las huellas de vínculos con otros que han sido significativos.

En último término, todo proceso de revinculación debe estar orientado por el interés superior del niño (ONU, 2010), de tal forma que la participación de éste y su familia debe ser permanente y activa. Mantener los vínculos no es un premio o un privilegio como consecuencia de una buena conducta, es un derecho que se debe garantizar para todo niño. Privar a un ser humano de sus vínculos es una de las vulneraciones más graves en estos contextos.

La intervención, desde ese punto de vista, apunta al egreso del niño de los contextos residenciales en situaciones donde las familias puedan reasumir el cuidado de los niños a través de un proceso de apoyo que les permita hacer frente de forma autónoma, y a la vez colectiva, de diversas situaciones que tensionan la vida familiar y los modos de cuidado y crianza de los niños. La revinculación del niño a su familia no se reduce al acto de egreso del niño de la residencia, sino que implica una preparación y posterior acompañamiento al grupo familiar en su adaptación posterior a la situación de separación. La tarea no es sólo generar egresos, sino más bien establecer garantías que permitan que los niños no vuelvan a sufrir una situación de separación.

Las políticas de infancia deben ser ante todo respetuosas de los niños, sus familias y comunidades. Estas no pueden prescindir de tener una comprensión de la experiencia de los niños que buscan proteger considerando sus vínculos, historias, trayectorias y contextos sociales, así como tampoco pueden pasar por alto las prácticas y discursos institucionales que hay entorno a la niñez y la familia.

Por lo mismo, el trabajo en este campo de intervención necesita reconocer que se constituye a través de un encuentro con vidas, cuyas historias muchas veces son reducidas al historial que se 
plasma, por ejemplo, en un informe social que se elabora a partir de un evento específico que es denominado vulneración de derechos y que excluye, en muchas ocasiones, que los sujetos que forman parte de estos contextos han estado expuestos históricamente a situaciones de vulnerabilidad y exclusión social.

Se trata de sujetos que no han tenido una experiencia recíproca de reconocimiento y que se perciben en permanente desventaja ante las instituciones que tienen la función de proteger y garantizar sus derechos. Cuestión a todas luces paradójica y que nos debe llevar a reflexionar en qué punto y bajo qué formas la institucionalidad actúa con arbitrariedad y violencia sobre determinados grupos de nuestra sociedad.

El desafío político en el ámbito de la internación de niños y niñas supone establecer un sistema de cuidado que genere modelos de trabajo y gestión que sean coherentes con los principios de la protección integral y la consideración de que el niño es, ante toda circunstancia, un sujeto de derecho que debe ser reconocido íntegramente y cuyos vínculos e identidad deben ser tratados con dignidad, particularmente, cuando se ve enfrentado a experiencias de vulneración que tienen como efecto la ruptura del lazo que lo une a su familia de origen.

\section{Referencias}

Aramburu, S. (2018). ¿Del enfoque tutelar al enfoque de derechos? Análisis de los marcos interpretativos de los actores legislativos en las principales leyes sobre familia. Recuperado el 10 noviembre de 2019, de https://www.sociedadpoliticaspublicas.cl/archivos/noveno/Infanc_Aramburu_Sofia.pdf

Anglade, J. (2005). Cómo la pobreza separa a los niños de sus padres. Espacio para la infancia, núm.24. Recuperado el 8 de julio de 2019, de: https://issuu.com/bernardvanleerfoundation/docs/ninos_carentes_de_cuidado_parental_alte rnativas_cu/24

Bustelo, E. (2011). El recreo de la infancia. Buenos Aires: Siglo XXI. 
Comité de los Derechos del Niño. (2018). Informe de la investigación relacionada en Chile en virtud del artículo 13 del Protocolo facultativo de la Convención sobre los Derechos del Niño relativo a un procedimiento de comunicaciones. Recuperado de:http://www.minjusticia.gob.cl/media/2018/07/Informe_del_Comit\%C3\%A9_de_la_Ni \%C3\%B1ez_de_ONU.pdf

Comisión Interamericana de Derechos Humanos (2013) Derecho del niños y la niña a la familia: cuidado alternativo: poniendo fin a la institucionalización en las Américas. Recuperado de: https://www.oas.org/es/cidh/infancia/docs/pdf/Informe-derecho-nino-a-familia.pdf

Cunill-Grau, N. (2014). La intersectorialidad en las nuevas políticas sociales: Un acercamiento analítico-conceptual. Gestión y política pública, 23(1), 5-46. Recuperado el 11 de noviembre de 2019, de http://www.scielo.org.mx/pdf/gpp/v23n1/v23n1a1.pdf

Delap, E. y Wedge, J. (2016). Directrices sobre la reintegración de niños, niñas y adolescentes. Recuperado el de julio de 2019, de: https://www.familyforeverychild.org/wpcontent/uploads/2016/08/Spanish_RG_v6_NNA.p $\underline{\mathrm{df}}$

Dolto, F. (2001). En el juego del deseo. México: Siglo XXI.

Eliacheff, C. (2002). Del niño rey al niño víctima. Violencia familiar e institucional. Buenos Aires: Nueva Visión.

Grinberg, J. (2016). Los Suárez y las instituciones del sistema de protección de la infancia: Un análisis sobre las formas contemporáneas de gobierno de las familias en contextos de desigualdad y pobreza. Revista Latinoamericana de Ciencias Sociales, Niñez y Juventud, 14 (1), pp. 631-643. Recuperado el 10 de diciembre de 2019, de: http://www.scielo.org.co/pdf/rlcs/v14n1/v14n1a44.pdf

Humphreys, D. (2011). La adopción de niños que han sido gravemente vulnerados en sus derechos. Maltrato Infantil, Reparación y Protección de la Infancia. Seminario llevado a cabo en Universidad Alberto Hurtado, Santiago, Chile.

Lebovici, S. Soulé, M. (1993). Tratado de psiquiatría del niño y el adolescente Volumen VIII. Madrid: Biblioteca Nueva.

Marchant, M. (2013). Vinculo y Memoria. Santiago: Cuarto Propio. 
Marchant, M; Morales C. (2016). Posesiones y pertenencias: Una reflexión sobre los objetos desde el pensamiento psicoanalítico. Congreso Chileno de Psicoanálisis. Congreso llevado a cabo en Sociedad Chilena de Psicoanálisis, Santiago, Chile.

Morales, C. (2015). En nombre de la protección. En Fernández, Lara, Oltra y Riquelme. Infancia, Familias y Estado. Santiago: Ediciones UCSH.

ONU (1989). Convención sobre los Derechos del Niño. Recuperado de: https://www.unicef.cl/web/informes/derechos_nino/convencion.pdf.

ONU (2009). Directrices sobre las modalidades alternativas de cuidado de los niños. Recuperado de: http://www.unicef.org/spanish/videoaudio/PDFs/100407-UNGA-Res-64-142.es.pdf

Relaf. (2011). Niñez y adolescencia institucionalizada: visibilización de graves violaciones de DDHH. Recuperado de https://www.relaf.org/biblioteca/Documento_agosto_2011_Relaf.pdf

Rojas, J. (2010) Historia de la Infancia en el Chile Republicano 1810-2010. Santiago, Chile: Ocho Libros.

Silva, D. \& Domínguez, P. (2017) Desinternar, sí. Pero ¿cómo? Controversias para comprender y transformar las propuestas institucionales de protección a la infancia y la adolescencia. Recuperado el 8 de julio de 2019, de: https://www.bibliotecaunicef.uy/doc_num.php?explnum_id=161

Spitz, R. (2006). El primer año de vida. México: FCE.

UNICEF. (2002). Internación de Niños ¿El Comienzo del Fin? Recuperado de: http://www.unicef.cl/web/wp-content/uploads/doc_wp/internacion.pdf

Universidad Diego Portales. (2018). Informe Anual sobre Derechos Humanos. Santiago

Villalta, C. (2013). Un campo de investigación. Las técnicas de gestión y los dispositivos jurídicoburocráticos destinados a la infancia pobre en Argentina. Civitas , 245-268.

Recepción: 29 de septiembre 2019

Última Revisión: 15 de diciembre 2019

Aceptación Final: 23 de diciembre 2019 